\title{
Singular Stress Field near Crack Front in Orthotropic Plate
}

\author{
Purong Jia*, Yongyong Suo and Gang Wang \\ Department of Engineering Mechanics, Northwestern Polytechnical University, Xi'an 710129, PR China \\ ${ }^{*}$ Corresponding author
}

\begin{abstract}
The analytic function of complex variable including a material parameter is analyzed fully. Typical crack model is considered to orthotropic materials. By constructing new stress function, the mechanic analysis for crack-tip singular stress field is carried out. Type boundary problems of partial equation are studied and the formulae for stress fields are derived. It is concluded that the stress fields in w-plane of the orthotropic material are similar to that of the isotropic material.
\end{abstract} field

Keywords—analytic function; orthotropic plate; singular stress

\section{INTRODUCTION}

Fracture mechanics of non-homogeneous materials or anisotropic materials has more applications in macroscopically heterogeneous materials. Prediction of crack initiation and propagation must be based on the fracture mechanics. Fiber-reinforced polymer matrix materials are the most typical composites and usually modeled as anisotropic materials at the macroscopic level [1]. The orthotropic plate may have been the base of composites in common use. So this paper focuses on the fracture of orthotropic materials. Singular stress fields at crack tip are mainly analyzed.

Linear elastic fracture mechanics (LEFM) has been found to be a very useful tool for design purposes and investigated in great detail for many engineering materials, whether isotropic or anisotropic [2]. The analysis of stresses near the crack tip holds an essential part of LEFM. The methods of elasticity are used to obtain stresses and displacements in cracked bodies [3]. The only viable method to solve stress-field problems in anisotropic composites is using complex analytic function theory, and the results have been reported $[4,5]$. But the general solution may have some weakness. So it is necessary to make up a new solution, and this is the paper purpose.

\section{COMPLEX FUNCTION REPRESENTATION}

It is well known that the basic complex variable $Z$ and its conjugate $\bar{Z}$ are defined as $(i=\sqrt{-1})$ :

$$
z=x+i y, \bar{z}=x-i y
$$

For the convenience of investigation, another complex variable $w$ is also introduced, that is:

$$
\begin{aligned}
& \qquad w=X+i Y=x+i h y, \bar{w}=X-i Y=x-i h y \\
& \text { and or , } X=x, Y=\text { hy }
\end{aligned}
$$

Where, the constant $h$ is real. The derivative relation must be as follows:

$$
\begin{gathered}
\frac{\partial w}{\partial X}=\frac{\partial w}{\partial x}=\frac{\partial \bar{w}}{\partial x}=\frac{\partial z}{\partial x}=\frac{\partial \bar{z}}{\partial x}=1, \frac{\partial z}{\partial y}=i, \frac{\partial \bar{z}}{\partial y}=-i \\
\frac{\partial w}{\partial Y}=\frac{\partial w}{h \partial y}=i, \frac{\partial \bar{w}}{\partial Y}=\frac{\partial \bar{w}}{h \partial y}=-i
\end{gathered}
$$

For the crack problem, rectangular and polar coordinates are shown in Figure I. The polar coordinate system centered at the crack tip may be adequate for local stress analysis. In terms of the polar coordinates (in z-plane and w-plane), the complex variables can be written as:

$$
\begin{aligned}
& z=x+i y=a+r \cos \theta+i r \sin \theta \\
& w=X+i Y=a+R \cos \Theta+i R \sin \Theta
\end{aligned}
$$

Thus, there are following relations:

$$
\begin{aligned}
R \cos \Theta= & r \cos \theta, R \sin \Theta=h r \sin \theta, \\
& \tan \Theta=h \tan \theta
\end{aligned}
$$

When $h=1$, then $\Theta=\theta, \quad R=r$. The w-plane is also reduced to z-plane.
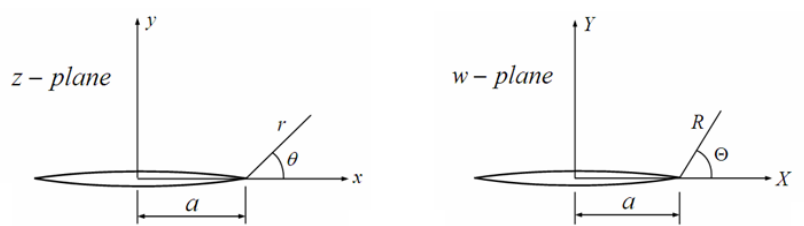

FIGURE I. SCHEME OF THE COORDINATES IN Z-PLANE AND W-PLANE

Consider an analytic function of complex variable, $\Psi=\Psi(w)$, and it can be expressed as: 
$\Psi(w)=\operatorname{Re} \Psi+i \operatorname{Im} \Psi=P+i Q=P(x, y)+i Q(x, y)$
Where, $P(\mathrm{x}, \mathrm{y})$ and $Q(\mathrm{x}, \mathrm{y})$ are real function. The derivative of $\Psi$ with respect to $W$ is given by

$$
\begin{aligned}
& \frac{\partial \Psi}{\partial X}=\frac{\partial \Psi}{\partial x}=\frac{d \Psi}{d w} \frac{\partial w}{\partial x}=\frac{d \Psi}{d w}=\Psi^{\prime}=\operatorname{Re} \Psi^{\prime}+i \operatorname{Im} \Psi^{\prime}=\frac{\partial P}{\partial x}+i \frac{\partial Q}{\partial x}=\frac{\partial P}{\partial X}+i \frac{\partial Q}{\partial X} \\
& \frac{\partial \Psi}{\partial Y}=\frac{1}{h} \frac{\partial \Psi}{\partial y}=\frac{1}{h} \frac{d \Psi}{d w} \frac{\partial w}{\partial y}=i \frac{d \Psi}{d w}=i \Psi^{\prime}=i \operatorname{Re} \Psi^{\prime}-\operatorname{Im} \Psi^{\prime}=\frac{\partial P}{\partial Y}+i \frac{\partial Q}{\partial Y}=\frac{1}{h}\left(\frac{\partial P}{\partial y}+i \frac{\partial Q}{\partial y}\right)
\end{aligned}
$$

Obviously, it is easy to derive the following differential equation:

$$
\begin{aligned}
& \frac{\partial P}{\partial X}=\frac{\partial P}{\partial x}=\frac{\partial Q}{\partial Y}=\frac{1}{h} \frac{\partial Q}{\partial y} \\
& \frac{\partial P}{\partial Y}=\frac{1}{h} \frac{\partial P}{\partial y}=-\frac{\partial Q}{\partial X}=-\frac{\partial Q}{\partial x}
\end{aligned}
$$

Which can be written as follows:

$$
\begin{aligned}
& \frac{\partial^{2} P}{\partial X^{2}}+\frac{\partial^{2} P}{\partial Y^{2}}=\frac{\partial^{2} P}{\partial x^{2}}+\frac{1}{h^{2}} \frac{\partial^{2} P}{\partial y^{2}}=0 \\
& \frac{\partial^{2} Q}{\partial X^{2}}+\frac{\partial^{2} Q}{\partial Y^{2}}=\frac{\partial^{2} Q}{\partial x^{2}}+\frac{1}{h^{2}} \frac{\partial^{2} Q}{\partial y^{2}}=0
\end{aligned}
$$

Therefore, two functions $\mathrm{P}$ and $\mathrm{Q}$ are harmonic functions in w-plane.

\section{BASIC EQUATIONS AND AIRY STRESS FUNCTION}

The plane stress state of composite sheets is common and very importance for the application. It is the key point to solve stress-field problems in orthotropic materials. The equilibrium equations are (body forces are absent):

$$
\begin{aligned}
& \frac{\partial \sigma_{x}}{\partial x}+\frac{\partial \tau_{x y}}{\partial y}=0 \\
& \frac{\partial \tau_{x y}}{\partial x}+\frac{\partial \sigma_{y}}{\partial y}=0
\end{aligned}
$$

The compatibility condition of strains must satisfy as follows:

$$
\frac{\partial^{2} \varepsilon_{x}}{\partial y^{2}}+\frac{\partial^{2} \varepsilon_{y}}{\partial x^{2}}=\frac{\partial^{2} \gamma_{x y}}{\partial x \partial y}
$$

Suppose the principal elastic directions coincide with the coordinate directions, and only consider the linear elastic strain-stress relations, then the constitutive equations for the orthotropic materials are given as (plane stress state):

$$
\begin{aligned}
& \varepsilon_{x}=\frac{\sigma_{x}}{E_{x}}-\frac{v_{x y} \sigma_{y}}{E_{x}} \\
& \varepsilon_{y}=\frac{\sigma_{y}}{E_{y}}-\frac{v_{x y} \sigma_{x}}{E_{x}} \\
& \gamma_{x y}=\frac{\tau_{x y}}{G_{x y}}
\end{aligned}
$$

It is well known that the Airy stress function $\mathrm{U}=\mathrm{U}(\mathrm{x}, \mathrm{y})$ is defined by:

$$
\sigma_{x}=\frac{\partial^{2} U}{\partial y^{2}}, \quad \sigma_{y}=\frac{\partial^{2} U}{\partial x^{2}}, \quad \tau_{x y}=-\frac{\partial^{2} U}{\partial x \partial y}
$$

The equilibrium equations in Eqs (6) can be satisfied. By using above relations, the governing equation of the Airy stress function, the compatibility equation (7) may be written as:

$$
\frac{\partial^{4} U}{\partial y^{4}}+B \frac{\partial^{4} U}{\partial x^{2} \partial y^{2}}+C \frac{\partial^{4} U}{\partial x^{4}}=0
$$

Where $B=\frac{E_{x}}{G_{x y}}-2 v_{x y}, C=\frac{E_{x}}{E_{y}}$.

The Airy stress function $U$ can be expressed by the real function, $P(\mathrm{x}, \mathrm{y})$ or $Q(\mathrm{x}, \mathrm{y})$. Consider an infinite plane with the crack along the $\mathrm{x}$-axis shown in Figure $\mathrm{I}$, and consider the problem of Mode I loading. The Airy stress function can be determined by the form:

$$
U=A_{1} \operatorname{Re} \Psi+A_{2} y \operatorname{Im} \Psi^{\prime}=A_{1} P+A_{2} y \frac{\partial Q}{\partial x}
$$

The derivatives of $\mathrm{U}$ with respect to $\mathrm{x}$ or $\mathrm{y}$ are given by: 


$$
\begin{aligned}
& \frac{\partial U}{\partial x}=A_{1} \frac{\partial P}{\partial x}+A_{2} y \frac{\partial^{2} Q}{\partial x^{2}} \quad, \quad \frac{\partial U}{\partial y}=A_{1} \frac{\partial P}{\partial y}+A_{2} \frac{\partial Q}{\partial x}+A_{2} y \frac{\partial^{2} Q}{\partial x \partial y} \\
& \frac{\partial^{2} U}{\partial x^{2}}=A_{1} \frac{\partial^{2} P}{\partial x^{2}}+A_{2} y \frac{\partial^{3} Q}{\partial x^{3}}, \quad \frac{\partial^{2} U}{\partial x \partial y}=A_{1} \frac{\partial^{2} P}{\partial x \partial y}+A_{2} \frac{\partial^{2} Q}{\partial x^{2}}+A_{2} y \frac{\partial^{3} Q}{\partial x^{2} \partial y} \\
& \frac{\partial^{2} U}{\partial y^{2}}=A_{1} \frac{\partial^{2} P}{\partial y^{2}}+2 A_{2} \frac{\partial^{2} Q}{\partial x \partial y}+A_{2} y \frac{\partial^{3} Q}{\partial x \partial y^{2}}
\end{aligned}
$$

On the basis of above equations, and by using Eq. (4) and Eq. (5), then Eq. (10) becomes:

$$
\left[\frac{A_{1}}{h}\left(h^{4}-B h^{2}+C\right)+2 A_{2}\left(B-2 h^{2}\right)\right] \frac{\partial^{4} Q}{\partial x^{3} \partial y}-\frac{A_{2}}{h^{2}}\left[h^{4}-B h^{2}+C\right] y \frac{\partial^{5} Q}{\partial x^{3} \partial y^{2}}=0
$$

Thus the solution must be followed by the characteristic equations, also reduced to:

$$
h^{4}-B h^{2}+C=0, \quad B-2 h^{2}=0
$$

The solution of the characteristic equation (14) is given as:

$$
h^{2}=\frac{B}{2}=\sqrt{C}=\frac{E_{x}}{2 G_{x y}}-v_{x y}=\sqrt{\frac{E_{x}}{E_{y}}}
$$

So the positive real root can be obtained, that is:

$$
h=\sqrt{\frac{E_{x}}{2 G_{x y}}-v_{x y}}=\sqrt[4]{\frac{E_{x}}{E_{y}}}
$$

For example, $E_{x}=120 G P a, E_{y}=30 G P a$, $G_{x y}=26 G P a, v_{x y}=0.3$, then giving: $h=1.4142$.

And again, $E_{x}=30 G P a \quad, \quad E_{y}=120 G P a$, $G_{x y}=26 G P a, v_{x y}=0.077$, then giving: $h=0.7071$.

So, the parameter $h$ can be $h>1$ also be $h<1$.

Substituting the derivative relation Eq. (12) into Eq. (9), the stresses can be expressed as:

$$
\begin{aligned}
& \sigma_{x}=A_{1} \frac{\partial^{2} P}{\partial y^{2}}+2 A_{2} \frac{\partial^{2} Q}{\partial x \partial y}+A_{2} y \frac{\partial^{3} Q}{\partial x \partial y^{2}} \\
& \sigma_{y}=A_{1} \frac{\partial^{2} P}{\partial x^{2}}+A_{2} y \frac{\partial^{3} Q}{\partial x^{3}} \\
& \tau_{x y}=-A_{1} \frac{\partial^{2} P}{\partial x \partial y}-A_{2} \frac{\partial^{2} Q}{\partial x^{2}}-A_{2} y \frac{\partial^{3} Q}{\partial x^{2} \partial y}
\end{aligned}
$$

Define $\Phi=\Phi(w)=\Psi^{\prime \prime}$. Thus, $\bar{\Phi}=\bar{\Phi}(\bar{w})=\bar{\Psi}^{\prime \prime}$. Then, some relations are given as:

$$
\begin{gathered}
\frac{\partial^{2} \Psi}{\partial x^{2}}=\Psi^{\prime \prime}=\Phi, \frac{\partial^{2} \Psi}{\partial y^{2}}=-h^{2} \Psi^{\prime \prime}=-h^{2} \Phi, \\
\frac{\partial^{2} \Psi}{\partial x \partial y}=i h \Psi^{\prime \prime}=i h \Phi
\end{gathered}
$$

$\frac{\partial^{2} Q}{\partial x^{2}}=\operatorname{Im} \Phi, \frac{\partial^{2} Q}{\partial y^{2}}=-h^{2} \operatorname{Im} \Phi, \frac{\partial^{2} Q}{\partial x \partial y}=h \operatorname{Re} \Phi$

Using above relations, the stresses in Eq. (17) can be written as: 


$$
\begin{aligned}
& \sigma_{x}=-A_{1} h^{2} \operatorname{Re} \Phi+2 A_{2} h \operatorname{Re} \Phi-A_{2} h^{2} y \frac{\partial \operatorname{Im} \Phi}{\partial x} \\
& \sigma_{y}=A_{1} \operatorname{Re} \Phi+A_{2} y \frac{\partial \operatorname{Im} \Phi}{\partial x} \\
& \tau_{x y}=A_{1} h \operatorname{Im} \Phi-A_{2} \operatorname{Im} \Phi-A_{2} h y \frac{\partial \operatorname{Re} \Phi}{\partial x}
\end{aligned}
$$

Evidently, the analytic function $\Phi$ can be as a new usual stress function.

\section{SOlution OF StREsses}

For Mode I crack problem, the plane with a line crack is subjected to symmetric loading $\tilde{\sigma}$ at infinity along y direction, and the stress boundary conditions are:

$$
\begin{gathered}
\sigma_{y}=\tau_{x y}=0 \text { at }|x|<a_{\text {and }} y=0 \text { (free crack surfaces) } \\
\sigma_{y}=\tilde{\sigma}, \tau_{x y}=0 \text { at }|x| \rightarrow \infty \text { and } y=0
\end{gathered}
$$

In order for the stress function to meet the preceding boundary value problem, the complex function can be selected as:

$$
\Phi=\operatorname{Re} \Phi+i \operatorname{Im} \Phi=\frac{w}{\sqrt{w^{2}-a^{2}}}=\operatorname{Re} \frac{w}{\sqrt{w^{2}-a^{2}}}+i \operatorname{Im} \frac{w}{\sqrt{w^{2}-a^{2}}}
$$

Substituting the stress function Eq. (21) into Eq. (18), and also using the boundary conditions Eqs. (19) and (20), the coefficients $A_{1}$ and $A_{2}$ can be derived as:

$$
A_{1}=\tilde{\sigma}, \quad A_{2}=h \tilde{\sigma}
$$

The stresses can be determined as follows:

$$
\begin{aligned}
& \sigma_{x}=\tilde{\sigma} \operatorname{Re} \frac{h^{2} w}{\sqrt{w^{2}-a^{2}}}+\tilde{\sigma} \operatorname{Im} \frac{a^{2} h^{3} y}{\left(w^{2}-a^{2}\right)^{3 / 2}} \\
& \sigma_{y}=\tilde{\sigma} \operatorname{Re} \frac{w}{\sqrt{w^{2}-a^{2}}}-\tilde{\sigma} \operatorname{Im} \frac{a^{2} h y}{\left(w^{2}-a^{2}\right)^{3 / 2}} \\
& \tau_{x y}=\tilde{\sigma} \operatorname{Re} \frac{a^{2} h^{2} y}{\left(w^{2}-a^{2}\right)^{3 / 2}}
\end{aligned}
$$

The complex variable $w$ can also be expressed in polar coordinates, that is:

$w-a=R[\exp (i \Theta)]=R[\cos \Theta+i \sin \Theta]=r[\cos \theta+i h \sin \theta]$
At the near-tip ( $r<<a$ ), the stresses of the orthotropic materials can be obtained (in z-plane):

$$
\begin{aligned}
\sigma_{x} & =\frac{K_{I} h^{2}}{\sqrt{2 r}}\left[\operatorname{Re} \frac{1}{\lambda}+\operatorname{Im} \frac{h \sin \theta}{2 \lambda^{3}}\right] \\
\sigma_{y} & =\frac{K_{I}}{\sqrt{2 r}}\left[\operatorname{Re} \frac{1}{\lambda}-\operatorname{Im} \frac{h \sin \theta}{2 \lambda^{3}}\right] \\
\tau_{x y} & =\frac{K_{I} h}{\sqrt{2 r}}\left[\operatorname{Re} \frac{h \sin \theta}{2 \lambda^{3}}\right]
\end{aligned}
$$

Where: $K_{I}=\tilde{\sigma} \sqrt{a}, \lambda=\sqrt{\cos \theta+i h \sin \theta}$. And also the stresses can be given as (in w-plane):

$$
\begin{aligned}
\sigma_{x} & =\frac{K_{I} h^{2}}{\sqrt{2 R}} \cos \frac{\Theta}{2}\left(1-\sin \frac{\Theta}{2} \sin \frac{3 \Theta}{2}\right) \\
\sigma_{y} & =\frac{K_{I}}{\sqrt{2 R}} \cos \frac{\Theta}{2}\left(1+\sin \frac{\Theta}{2} \sin \frac{3 \Theta}{2}\right) \\
\tau_{x y} & =\frac{K_{I} h}{\sqrt{2 R}} \cos \frac{\Theta}{2}\left(\sin \frac{\Theta}{2} \cos \frac{3 \Theta}{2}\right)
\end{aligned}
$$

For the isotropic materials ( $h=1$ ), the stresses can be reduced to:

$$
\begin{aligned}
\sigma_{x} & =\frac{K_{I}}{\sqrt{2 r}} \cos \frac{\theta}{2}\left(1-\sin \frac{\theta}{2} \sin \frac{3 \theta}{2}\right) \\
\sigma_{y} & =\frac{K_{I}}{\sqrt{2 r}} \cos \frac{\theta}{2}\left(1+\sin \frac{\theta}{2} \sin \frac{3 \theta}{2}\right) \\
\tau_{x y} & =\frac{K_{I}}{\sqrt{2 r}} \cos \frac{\theta}{2} \sin \frac{\theta}{2} \cos \frac{3 \theta}{2}
\end{aligned}
$$

Obviously, the stress fields in w-plane for the orthotropic material are similar to that of the isotropic material. Nevertheless, the stress strength coefficients are not uniform $(h \neq 1)$.

\section{ACKNOWLEDGEMENTS}

The authors acknowledge the financial support of the Natural Science Foundation of China (NSFC Grant No. 51475372).

\section{REFERENCES}

[1] Klaus Friedrich: Application of fracture mechanics to composite materials. Elsevier Sci. Pub. B.V., (1989)

[2] G.C. Sih: Mechanics of fracture initiation and propagation. Kluwer Academic Publishers, (1991). 
[3] C.T. Sun: Fracture mechanics. Elsevier Inc., (2012).

[4] M. Fakoor, N.M. Khansari. Mixed mode I/II fracture criterion for orthotropic materials based on damage zone properties. Eng Fract Mech 2016; 153: 407-420.

[5] M. Fakoor. Augmented strain energy release rate (ASER): A novel approach for investigation of mixed-mode I/II fracture of composite materials. Eng Fract Mech 2017; 179: 177-189. 